\title{
A Comparative Plasmonic Study of Nanoporous and Evaporated Gold Films
}

\author{
Stefanie Ahl • Petra J. Cameron • Jing Liu • \\ Wolfgang Knoll • Jonah Erlebacher • Fang Yu
}

Received: 1 August 2007 / Accepted: 14 November 2007 / Published online: 8 January 2008

(C) Springer Science + Business Media, LLC 2007

\begin{abstract}
Previously, we have reported that nanoporous gold (NPG) films prepared by a chemical dealloying method have distinctive plasmonic properties, i.e., they can simultaneously support localized and propagating surface plasmon resonance modes (1-SPR and p-SPR, respectively). In this study, the plasmonic properties of NPG are quantified through direct comparison with thermally evaporated gold (EG) films. Cyclic voltammetry
\end{abstract}

Electronic supplementary material The online version of this article (doi:10.1007/s11468-007-9048-5) contains supplementary material, which is available to authorized users.

\footnotetext{
S. Ahl $(\bowtie) \cdot$ P. J. Cameron · J. Liu • W. Knoll • F. Yu $(\bowtie)$

Department of Materials Science,

Max Planck Institute for Polymer Research,

Ackermannweg 10,

55128 Mainz, Germany

e-mail: ahl@mpip-mainz.mpg.de

e-mail: fangy@stanford.edu

W. Knoll

e-mail: knoll@mpip-mainz.mpg.de

J. Erlebacher

Department of Materials Science and Engineering,

Johns Hopkins University,

Baltimore, MD 21218, USA

Present address:

P. J. Cameron

Department of Chemistry, University of Bath,

Bath BA2 2TB, UK

J. Liu

Siemens X-Ray Vacuum Technology Ltd.,

Wuxi 214028, People's Republic of China

F. Yu

Department of Chemistry, Stanford University,

Stanford, CA 94305, USA
}

and electrochemical impedance spectroscopy experiments reveal that the NPG films have 4-8.5 times more accessible surface area than EG films. Assemblies of streptavidinlatex beads generate p-SPR responses on both NPG and EG films that correlate well with the bead density obtained from scanning electron microscopy (SEM) images. A layerby-layer assembly experiment on NPG involving biotinylated anti-avidin IgG and avidin, studied by 1-SPR and SEM, shows that the 1-SPR signal is directly linked to the accessibility of the interior of the NPG porosity, an adjustable experimental parameter that can be set by the dealloying condition and time.

Keywords Surface plasmon resonance - Alloys .

Layer deposition · Spectroscopy · Nanoporous gold ·

Gold films $\cdot$ Electrochemistry

\section{Introduction}

There is continued interest [1-6] in discovering nanoporous metallic materials for sensing, catalysis, and filtration purposes. Recently, we reported a facile approach to the fabrication of large area, thin, metallic nanoporous gold (NPG) membranes. NPG is formed by a spontaneous pattern-forming instability during the chemical etching of silver from gold/silver alloys, the so-called dealloying process. An analytical atomistic model presented earlier [7] has clarified the underlying physics of porosity evolution during the dealloying. In contrast to the majority of porous metallic nanostructures that are generally controlled aggregates of metallic nanoparticles [8], NPG retains a spatially coherent crystal lattice that extends to the scale of the original grain structure of the non-porous alloy. Extending the dealloying time can significantly coarsen the 
grain structure in NPG [9] as a result of gold atom surface diffusion driven by surface-energy reduction. Therefore, the porosity may be conveniently adjusted from less than $10 \mathrm{~nm}$ up to the macroscopic scale of the original starting material [9].

NPG with $\sim 15 \mathrm{~nm}$ pores itself is not gold-colored (it is a dark bronze), and elucidation of its optical properties, as they correlate to its nanoscale morphology, is a clear research direction. Indeed, in our recent report, we have found intriguing plasmonic properties of NPG that point to the utility of this material in (bio)sensing contexts [10]. Specifically, NPG was found to simultaneously support both propagating surface plasmon resonance (p-SPR) modes and localized surface plasmon resonances (1-SPR). Propagating surface plasmons are evanescent electromagnetic waves bound to flat metal-dielectric interfaces that arise from oscillations of the conduction electrons in the metal. If surface plasmons are confined to periodic, colloidal, or other nanosystems, localized optical modes are observed. These optical modes lead to highly localized electromagnetic fields outside the particles. Both p-SPR and 1-SPR are sensitive to local refractive index changes that occur if target analytes bind to the metal film or nanoparticles. Surface refractive index sensors have an inherent advantage over fluorescence optical biosensors that require a chromophoric group or other luminescence label to transduce the binding event.

The compatibility of NPG with these two state-of-art sensing methods immediately opens up numerous sensing opportunities for NPG. In our research, we have discovered that the use of NPG is not a reiteration of "another kind of metal" for plasmonic sensing. There are unique properties linked to the porosity. First, NPG provides a greatly enhanced surface-to-volume ratio, which is beneficial for better reaction efficiency and detection sensitivity. Second, porous materials in general are particularly useful as separation media owing to their size-selectivity in chromatographical isolation, adsorption, catalysis, etc. The nanosized porous structure strongly affects the sequential build-up of functional supramolecular architectures on substrates that is inherent in the sensing process itself. The diffusion of molecules to the NPG interior is hindered if the pore size is comparable with the molecular dimension; this "molecular sieve" effect can be used for size-selective adsorption and examination of diffusional kinetics through nanoscale channels. Third, by simultaneously supporting p-SPR and 1-SPR modes, NPG effectively probes the interfacial refractive index variations at the drastically different scales associated with each plasmonic mode. Fourth and finally, for supported membrane applications, NPG can double as an effective water reservoir and conductive substrate for electrochemical investigations.

This report presents a quantitative side-by-side comparison of NPG films with evaporated gold (EG) films. We discuss the following experiments. Firstly, an electrochemical oxidation experiment was conducted to study the area ratio of NPG compared to EG films. Secondly, p-SPR modes were employed to monitor the growth of a biological multilayer involving the assembly of streptavidin-coated latex beads, which generate quantifiable signals recorded by two methods, i.e., by p-SPR and by scanning electron microscopy (SEM) images. Finally, a layer-by-layer (LbL) assembling experiment on NPG involving biotinylated antiavidin IgG and avidin was performed and monitored by both 1-SPR and SEM.

\section{Experimental}

NPG fabrication

NPG leaf membranes were produced by dealloying $\sim 100 \mathrm{~nm}$ thick, 12-carat white-gold decorative leaf $(\mathrm{Ag} / \mathrm{Au}$ 1:1 ratio by weight) in $70 \%$ nitric acid for certain time period [9] (etching time) and were bonded onto LaSFN9 glass slides (Schott Glass, $n=1.85$ at $\lambda=632.8 \mathrm{~nm}$ ) pre-functionalized by 3 -mercaptopropyltrimethoxysilane to promote adhesion and stability [11] of the metal membranes under aqueous buffer conditions.

By applying a potential during the residence time of the NPG in the nitric acid, the dealloying process happened within seconds, and very small pore sizes $(\sim 5 \mathrm{~nm})$ could be achieved.

\section{Electrochemical experiments}

Electrochemical studies including cyclic voltammetry (CV) and electrochemical impedance spectroscopy (EIS) were carried out using an Autolab PGSTAT 30 (Ecochemie) with an internal frequency response analyzer. The gold films (nanoporous or evaporated) supported on a glass slide were used as the working electrodes. The electrochemical Teflon cell was tightly clamped to the gold slide with an o-ring, which defined an active surface area of $0.77 \mathrm{~cm}^{2}$. A platinum coil counter electrode and a silver/silver chloride reference electrode (World Precision Instruments, DriRef-2) completed the cell. The electrolyte was either $0.1 \mathrm{M} \mathrm{KNO}_{3}$ or $1 \mathrm{M}$ sulfuric acid. Impedance spectra were fit using the modeling program of the Ecochemie FRA software. The equivalent circuit was

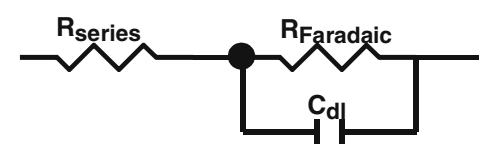

where $R \mathrm{~s}$ was the series resistance, $R_{\mathrm{F}}$ the Faradaic resistance, and $C_{\mathrm{dl}}$ the double layer capacitance. The 
impedance spectra reported were taken at $0.3 \mathrm{~V}$ vs silver/silver chloride in $1 \mathrm{M}$ sulfuric acid; at this potential, no Faradaic peaks were seen in the $\mathrm{CV}$ results.

\section{SPR set-up}

For the p-SPR measurement, a home-built surface plasmon resonance spectrometer based on the Kretschmann configuration was used, which has been described in detail elsewhere [12]. Briefly, a linearly polarized, frequencymodulated laser source (HeNe, $10 \mathrm{~mW}, \lambda=1152 \mathrm{~nm}$; Laser 2000) illuminates the gold film through a right-angled LaSFN9 glass prism. The reflected laser intensity is measured by a photo-diode detector connected to a lockin amplifier. The sample (prism, gold slide, and quartz flow cell) and the detector are mounted to two co-axial goniometers, respectively. L-SPR was measured simultaneously using a fiber optic spectrometer (USB2000, Ocean Optics) equipped with a reflection probe (R400-7, Ocean Optics) and a light source (HL-2000, Ocean Optics). The 1SPR experiments measured the reflection spectrum of the gold sample through a quartz window of a flow-cell clamped to the sample. The reflection spectra $(R)$ shown in the figures were measured by taking the ratio between the raw detector spectrum for the measurement sample $\left(I_{\text {sample }}\right)$ and the raw detector spectrum for the reference sample $\left(I_{\text {ref }}\right)$. The dark signal $\left(I_{\text {dark }}\right)$ was subtracted from the raw spectra:

$R=\log _{10}\left(I_{\text {sample }}-I_{\text {dark }} / I_{\text {ref }}-I_{\text {dark }}\right)$
Protein multilayer formation

Gold (nanoporous or evaporated) films were first functionalized by a biotinylated self-assembled monolayer containing $\sim 10 \%$ of the biotin groups [13]. Next, a streptavidin layer was formed from a $1-\mu \mathrm{M}$ streptavidin (Roche Diagnostics) solution followed by a biotinylated $\mathrm{IgG}$ antibody (Molecular Probes) layer from a $1-\mu \mathrm{M}$ IgG solution. Finally, a 1\%(w/v) streptavidin-doped polystyrene latex (Roche Diagnostics) solution was introduced into the flow-cell. The streptavidin-modified polystyrene latex beads were obtained from Roche Diagnostics and had an average radius of $r=56 \mathrm{~nm}$; the refractive index was $n=$ 1.56 at $\lambda=1152 \mathrm{~nm}$. They were diluted in phosphate buffer with $0.01 \%(w / t)$ Tween $-20^{\mathrm{R}}$.

A fast-growing LbL assembly system based on avidin (Sigma) and biotinylated anti-avidin antibody (BAA) was also employed as model system. Gold (nanoporous or evaporated) films were first functionalized by the biotinylated SAMs. Then, avidin/BAA solutions (both $1 \mu \mathrm{M}$ in PBS buffer with $0.01 \%(w / t)$ Tween- $\left.20^{\mathrm{R}}\right)$ were alternated in the flow cell, leading to the stepwise formation of an avidin/BAA multilayer. The use of BAA significantly enhanced the recognition probability to avidin and accelerated the layer thickness increase (Supporting Materials 1).

Phosphate-buffered saline (PBS) with $0.01 \%(w / t)$ Tween-20 ${ }^{\mathrm{R}}$ was used as solvent for all the sample solutions. For each layer-forming step, the binding was monitored kinetically and terminated by a PBS buffer rinse once equilibrium was reached.
Fig. 1 The cyclic voltammogram for a 15-min-etched NPG sample is depicted. Gold oxides were formed at positive potentials and reduced at less positive potentials. The sweep rate was $100 \mathrm{mV} \mathrm{s}^{-1}$, and the electrolyte was $1 \mathrm{M}$ $\mathrm{H}_{2} \mathrm{SO}_{4}$. The current in milli amperes is plotted vs the potential in volts with respect to the silver/ silver chloride reference electrode

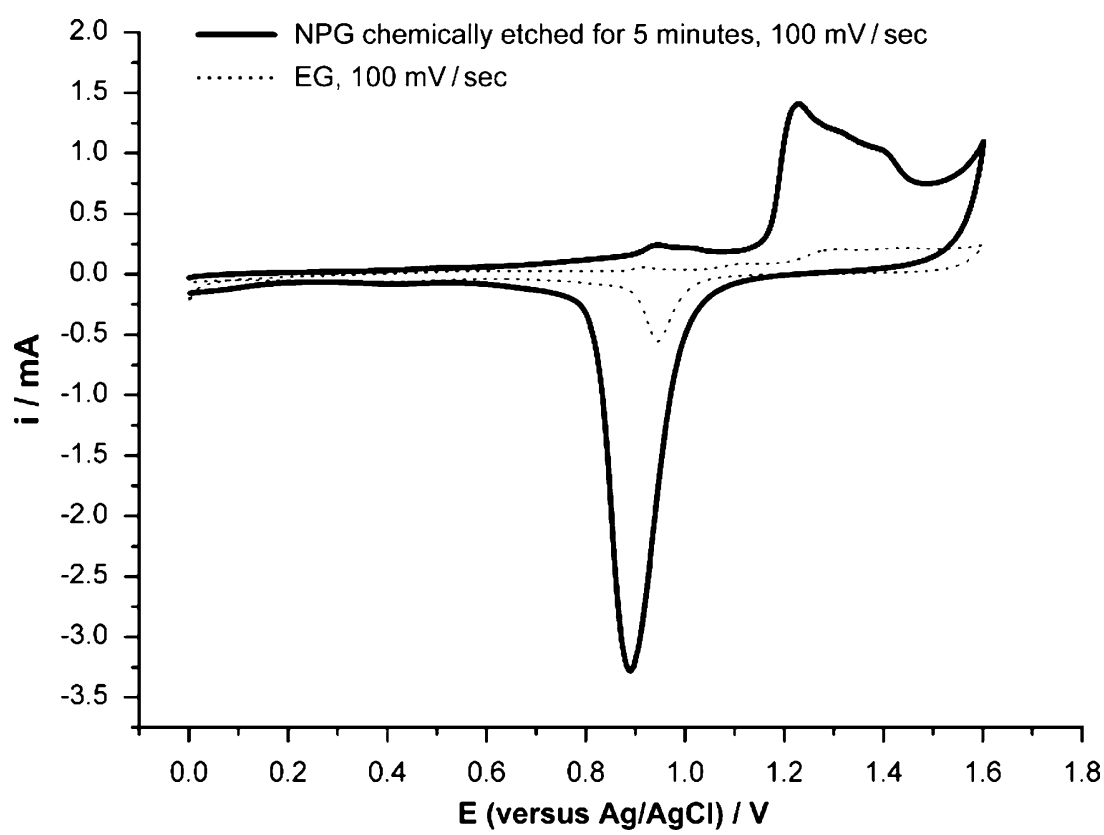




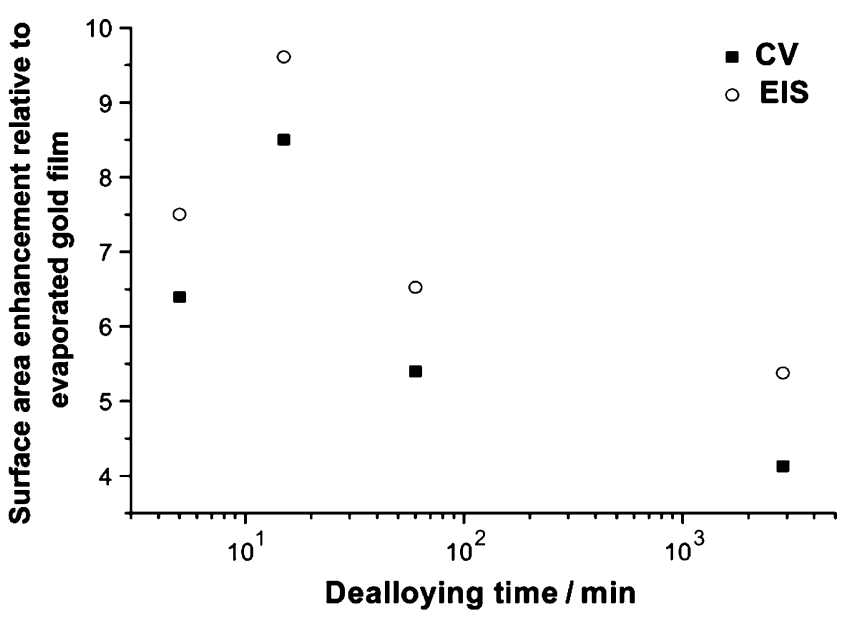

Fig. $2 \mathrm{CV}$ and EIS measurements reveal a surface area enhancement for the chemically dealloyed NPG samples compared to EG

\section{Results and discussions}

Electrochemical characterization

The surface area of the NPG was characterized by CV and by EIS. In the $\mathrm{CV}$, an oxide layer was formed at potentials positive of $1 \mathrm{~V}$ during the anodic scans in $1 \mathrm{M}$ sulfuric acid. On the subsequent cathodic scans, the oxide layer was stripped and a sharp peak was seen in the voltammograms [14] (Fig. 1). The area under the reduction peak is proportional to the charge needed to reduce the gold oxide monolayer and is related to the surface area of the gold film [15].

Electrochemical impedance spectroscopy was used to obtain values for the double layer capacitance of the NPG films [16]. Provided the distance of the closest approach of the counter ions is sufficiently small, the double layer capacitance will be proportional to the surface area of the gold films.

Figure 2 shows the variation in the surface area of the NPG with respect to chemical dealloying time, measured by both CV and EIS. In all cases, it is assumed that the EG films had a surface area of 1 , and the numbers shown refer to the surface increase relative to these films. All NPG films show an enhanced surface area relative to EG.

The NPG films chemically etched under free corrosion for $5 \mathrm{~min}$ have surface areas six times larger than the EG. The largest surface increase ( 10 -fold $)$ was seen for the sample chemically etched for $15 \mathrm{~min}$. Chemical dealloying

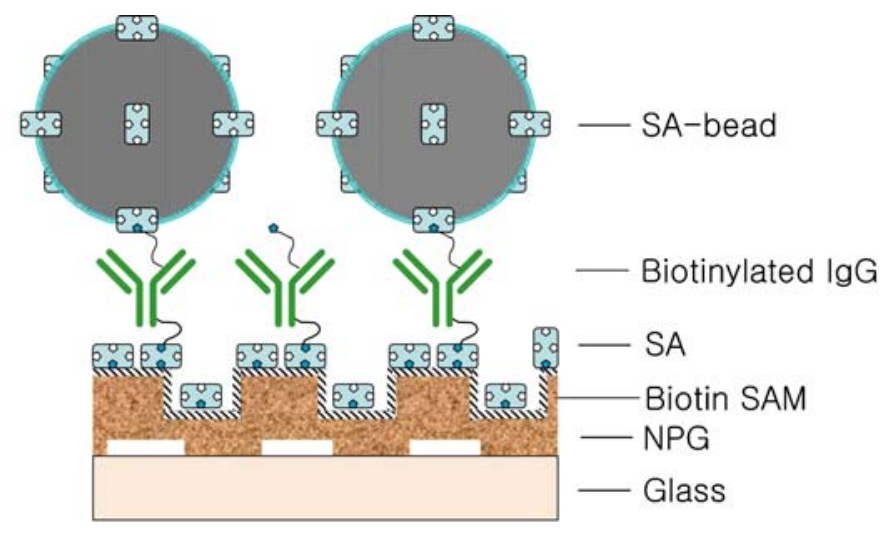

a

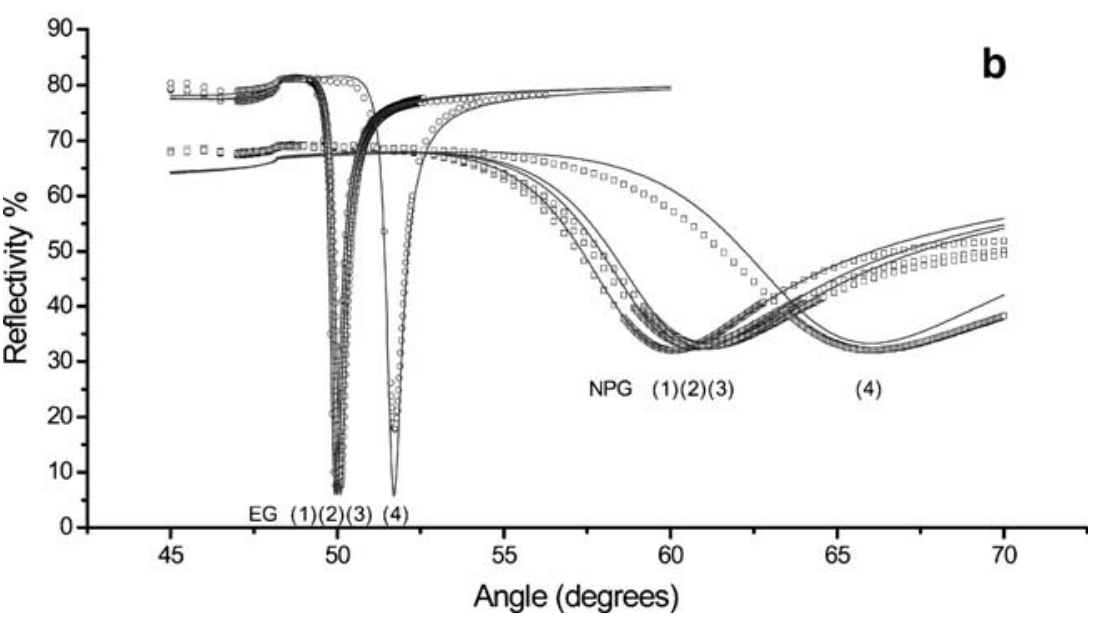

Fig. 3 Multi-layer formation on NPG-a model system: a A schematic representation on which the measurements are based. b p-SPR $(\lambda=$ $1152 \mathrm{~nm}$ ) measurements in buffer condition monitoring of streptavi$\mathrm{din} /$ biotinylated IgG bindings on biotin SAM modified $100 \mathrm{~nm}$ thick NPG membrane and $50 \mathrm{~nm}$ evaporated gold film surface, respectively.
Additionally polystyrene latex bead were deposited on top of the supramolecular architecture. For both substrates, curve (1) is for SAM modified sample, curve (2) for after streptavidin binding, curve (3) for after biotinylated IgG binding; and finally curve (4) represents the latex bead binding result 

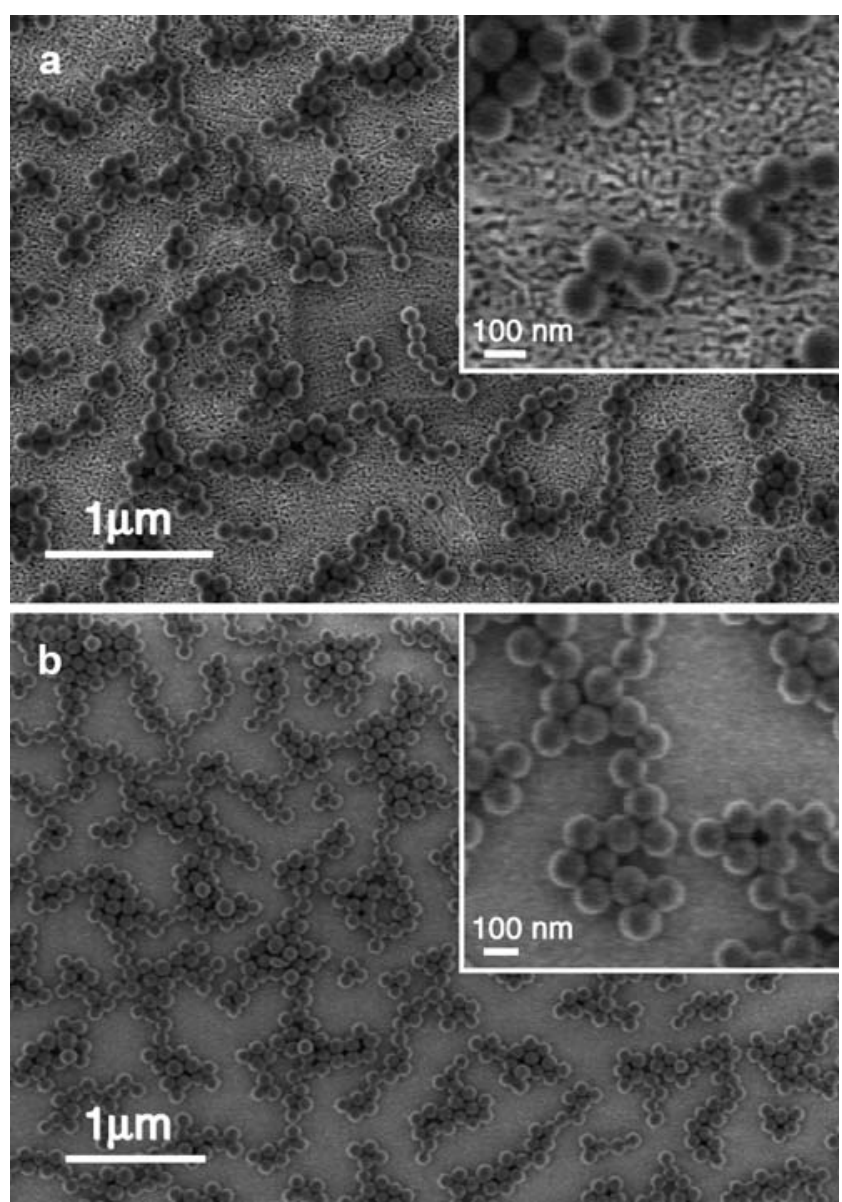

Fig. 4 Scanning electron images of latex beads assembled on a NPG sample and $\mathbf{b}$ evaporated gold sample

times longer than 15 min led to a decrease in the surface area enhancement. This decrease was thought to correlate to the collapse of the porous 3D structure into a 2D structure, which can be seen in the lateral view of the SEM images [9]. The surface enhancement measured by CV agrees broadly with that measured by EIS; in addition, the same trends are seen by both methods with respect to increasing chemical dealloying time.

\section{Streptavidin-doped bead density}

A multilayer system was built on NPG substrates that were etched for $5 \mathrm{~min}$. The streptavidin beads, with an average diameter of $d=112 \mathrm{~nm}$, were chosen because they generate a significant SPR signal and the beads can be easily visualized by SEM. Therefore, the bead density could be quantified by both methods, and a comparison could be made in order to verify the applicability of NPG as a SPR supporting substrate. As shown in the schematics (Fig. 3a), two protein layers, streptavidin and biotinylated $\mathrm{IgG}$, were pre-deposited on NPG (5 min etched) and EG films, respectively, to generate a dense layer of biotin functional groups. As reported in our previous paper [10], twice as much streptavidin binding occurred on NPG compared to EG. Only double the coverage was measured because streptavadin is a large molecule, and due to the steric hindrance, only a fraction of the NPG area was accessible to it. The subsequent biotinylated $\operatorname{IgG}$ binding amount was comparable for both films, which can also be attributed to the size selectivity of the NPG surface.

Furthermore, we should note that a near-infrared laser with a wavelength of $\lambda=1152 \mathrm{~nm}$ was used to compensate for the heavy damping loss of SPR propagation on NPG surface, which has also been discussed in our previous report [10].

The bead binding generated a significant SPR minimum shift for both the NPG and the EG films. In this study, we consider a virtual 'layer' composed of polystyrene beads and water, with a constant thickness given by the bead diameter $(112 \mathrm{~nm})$ and a variable refractive index representing the change in bead coverage. The averaged dielectric constant $\varepsilon_{\text {average }}$ is assumed to be the weighted average of each component:

$\varepsilon_{\text {average }}=\varepsilon_{\text {water }}(1-\chi)+\varepsilon_{\text {bead }} \chi$,

where $\varepsilon_{\text {water }}$ and $\varepsilon_{\text {bead }}$ are the dielectric constants of water and bead, respectively, and $\chi$ is the volume ratio of the beads. During binding, the increase of $\chi$ leads to the increase of $\varepsilon_{\text {average. }}$. Fresnel fits were conducted to give the optical thickness change before and after bead binding. Because the thickness of the 'layer' is fixed, $\varepsilon_{\text {average }}$ and $\chi$ can be determined by Fresnel fits. We made two simplifications for this calculation process. First, we considered $\varepsilon_{\text {average }}$ to be constant normal to the surface of the layer, i.e., we treat the spherical bead as cylinders. Due to the exponential decay of the SPR wave, this simplification will introduce a certain error in the output. However, because we used a $\lambda=1152 \mathrm{~nm}$ laser, the evanescent wave of SPR has a 1/e decay length of 350-500 nm, inside which a $112 \mathrm{~nm}$ thick layer can be treated as a relatively 'thin' layer. Moreover, the overestimation and underestimation caused by top, middle, and bottom part of the spherical bead are, to some extent, mutually cancelled. Second, as can be seen in the Fig. 3b, the binding of the beads not only shifts the SPR curve but also increases the minimum reflectivity (in EG's case) or

Table 1 Latex bead coverage results obtained by p-SPR and SEM on NPG (two substrates shown) and flat gold samples

\begin{tabular}{llllll}
\hline $\begin{array}{l}\text { Gold } \\
\text { film }\end{array}$ & $\varepsilon_{\text {bead }}$ & $\varepsilon_{\text {water }}$ & $\varepsilon_{\text {ave }}$ & $\begin{array}{l}\text { Volume ratio } \\
(\text { SPR; } \%)\end{array}$ & $\begin{array}{l}\text { Volume ratio } \\
\text { (SEM) }\end{array}$ \\
\hline NPG & 2.434 & 1.752 & 1.94 & 27.6 & $29 \pm 2 \%$ \\
NPG & & & 1.892 & 20.5 & $21 \pm 2 \%$ \\
EG & & & 1.945 & 28.3 & $27 \pm 2 \%$ \\
\hline
\end{tabular}


broadens the dip width (in the NPG case). This is primarily due to the enhanced scattering loss of the SPR field attributed to the new surface 'roughness' introduced by the beads, which have a size comparable to the wavelength of the light and have a refractive index significantly higher than the surrounding medium, i.e., water. Fortunately, this scattering loss, within certain range, should not significantly influence the angular position of the SPR minimum (see Supporting Materials 2). Therefore, the Fresnel fit in the bead binding step was only targeted to fit the SPR minimum position, i.e., only the real part of $\varepsilon_{\text {average }}$ was adjusted.

The bead-modified surfaces were also imaged by SEM (cf. Fig. 4). One can see that on both surfaces, the beads tend to form similar patterns, i.e., they adhere to each other to form chains and islands and there seems to be a certain spacing distance between islands. Although concentrated bead suspensions were used, the bead coverage

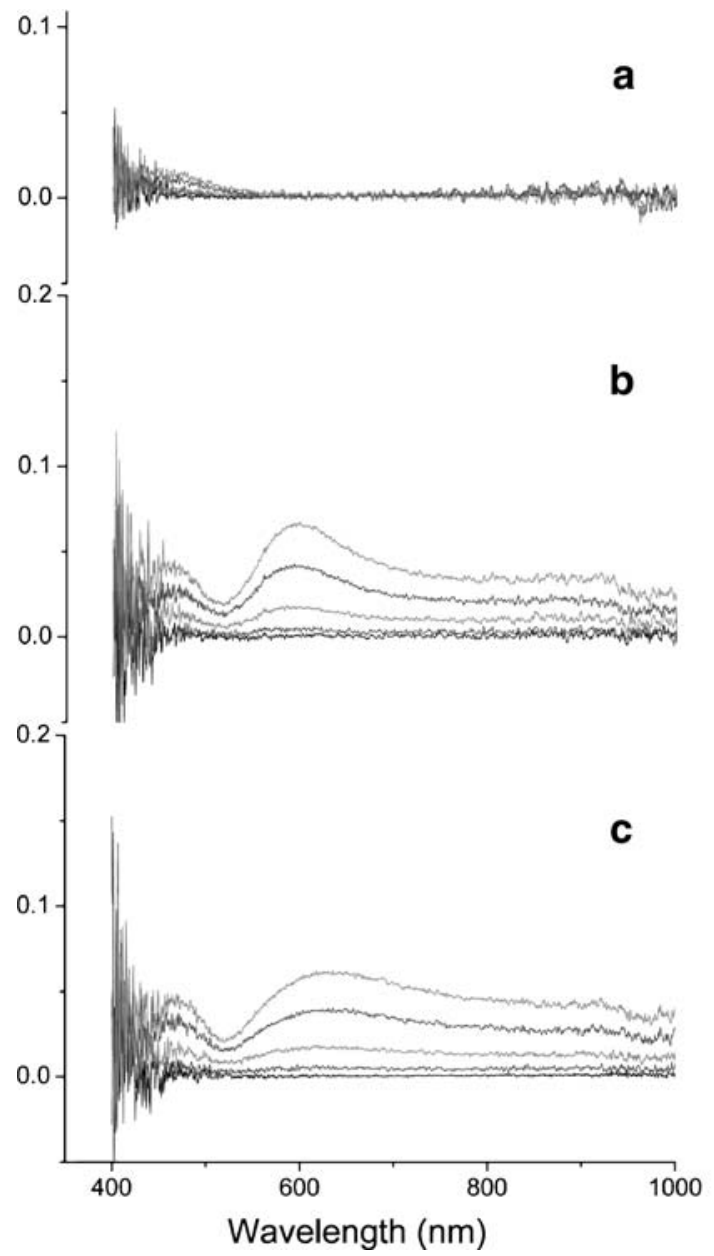

Fig. 5 Relative reflection absorbance spectra of a a flat evaporated gold film, b a 5-min dealloyed NPG, and $\mathbf{c}$ a 24-h dealloyed NPG, upon exposure to a series of glycerol solutions $(5,15,35$, and $60 \%)$. The relative reflection absorbance spectra of $\mathbf{d}$ a flat evaporated gold film, e a 5-min dealloyed NPG, f a 24-h dealloyed NPG, during LbL deposition of 3.5 Avidin/BAA double-layers. The gold (evaporated or was in the range of $20-30 \%$. Both SPR and SEM data provide the bead coverage results presented in Table 1 . For both NPG and EG, SPR and SEM give comparable results for the coverage. This leads to the conclusion that NPG can be used as a substrate that supports propagating SPR, and this p-SPR can be used to quantify the binding that happens outside, on the surface of, the nanopores of NPG.

\section{LbL deposition study}

Further refractive index studies on different samples were carried out using the same optical setup. The refractive index was tuned either in the bulk by glycerol solutions or at the interface by multilayer deposition (Fig. 5). The refractive index change was experimentally compared for

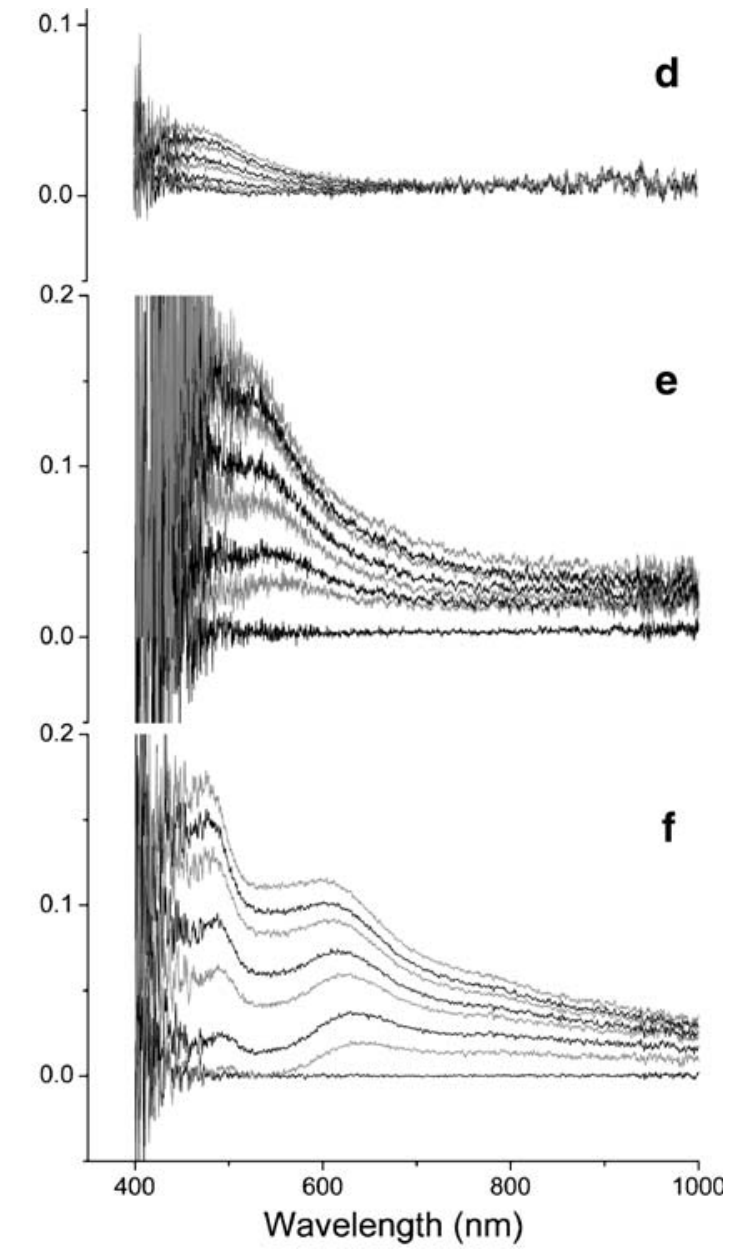

nanoporous) film was pre-functionalized by a biotinylated self assembled monolayer containing $\sim 10 \%$ functional biotin groups. Then an avidin layer was formed followed by a biotinylated antiavidin antibody layer. The spectra were recorded after the respective protein layer was complete 
two NPG substrates possessing different pore sizes (5min etched NPG as NPG5 min, 24-h etched NPG as NPG24h) and for an EG film. After injecting various glycerol concentrations (from 5 to $60 \%$ ), the EG film showed only a limited absorbance at a wavelength range $(\sim 400-500 \mathrm{~nm})$ near the noise range of the white-light lamp serving as the excitation source. This absorbance also seen, even more pronouncedly, for both NPG samples, which still lacks a satisfying explanation. In addition to that, both NPG samples soaked in glycerol solutions that permeate into the pores exhibited significant changes in their absorbance spectra $(\sim 530-800 \mathrm{~nm})$. Exposure to higher glycerol concentrations caused an obvious increase in the peak intensity and a slight red shift of the peak position; both are strong evidence for the excitation of localized SPR [17]. The peak wavelength is slightly higher for NPG24h $(\lambda=625 \mathrm{~nm})$ than for NPG5min $(\lambda=590 \mathrm{~nm})$ due to the larger nanosized features in NPG24h.

A LbL assembly system (different from the LbL system used in our previous work) [18] was employed in this study to dynamically and quickly alter the interfacial refractive index. The use of biotinylated anti-avidin IgG and avidin formed a mutual recognition system and greatly enhanced the thickness of each double layer $(d \sim 9.3 \mathrm{~nm}$ with an assumed refractive index of $n=1.45$ ). This fastgrowing LbL system has been studied extensively in our group [19].

In comparison to the $\mathrm{LbL}$ system used in our previous report [10], which involved oppositely charged dendrimers with a molecular weight of $\sim 35 \mathrm{kDa}$, the current system has a molecular size that can cause dramatically different binding pattern on NPGs with different pore sizes. As shown in the SEM pictures (cf. Fig. 6), the protein layers on NPG5min have a distinct interface to the NPG film, meaning that the proteins are mostly binding onto the NPG. In contrast, in the NPG24h image, it is hard to tell where the protein layer starts, which clearly indicates binding of the proteins inside the pores.

The SEM observation is confirmed by the 1-SPR results. For clarity, we only show the 3.5 double-layerdepositing process (cf. Fig. 5d-f). On the EG surface, there is a growing peak between $430-600 \mathrm{~nm}$. This turned out to be a typical reflective interference phenomenon caused by the deposited dielectric layer, as manifested by the later periodical oscillation in reflectivity as the protein layer grew (data not shown). A similar interference pattern was observed on NPG5m. In the NPG24h curves, we saw not only a similar interference pattern but also a distinctive peak at $625 \mathrm{~nm}$, exactly matching its peak induced by glycerol irrigation. In contrast, the curves of NPG5min sample are free of the peak at its characteristic wavelength, $590 \mathrm{~nm}$. This observation is in agreement with the
SEM image that only proteins binding into the nanopores can modify the dielectric environment and thus alters 1SPR signal. The fast saturation of 1-SPR signal is also in agreement with the fact that $1-S P R$ has very short evanescent decay $(\sim 10-20 \mathrm{~nm})$.

\section{Conclusion}

A series of comparative experiments between NPG and EG samples are reported. The electrochemical experiments indicate a nearly one order-of-magnitude enhancement of surface area for NPG over EG. The streptavidin-doped bead binding experiment shows the utility of NPG for quantitative p-SPR measurement. Finally, the LbL assembly experiment reveals the different binding mode of large biomolecules (e.g., avidin and IgG) to NPGs possessing different pore sizes, a hypothesis supported by both 1-SPR and SEM observation. Our observations will help to further understand the character of NPG as a novel plasmonic substrate for wider applications.
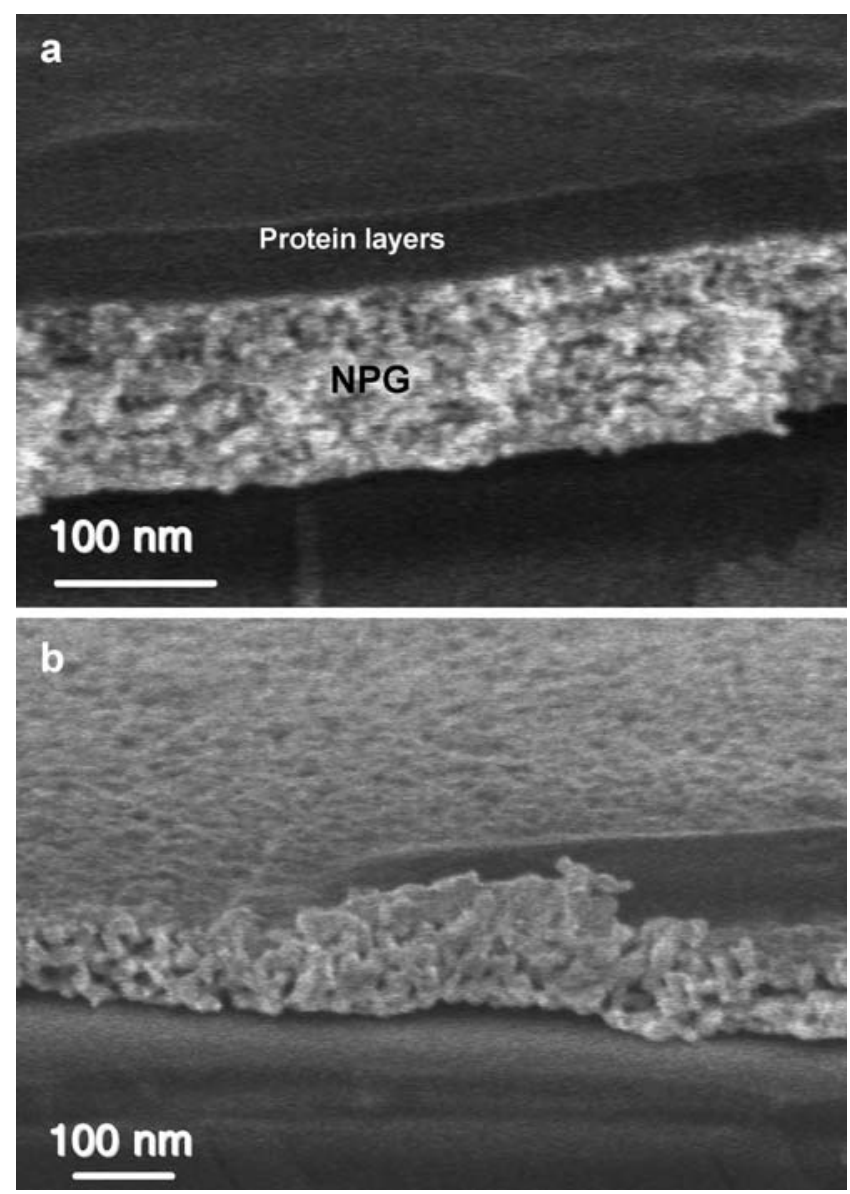

Fig. 6 SEM images of a a 5-min-etched NPG after LbL deposition of 18 double layers of avidin/BAA. b A 24-h-etched NPG after deposition of 11 double layers of avidin/BAA 
Acknowledgement The authors thank Jianjun Wang and Gunnar Glasser (MPIP) for SEM measurements. Support from the NSF under grant CTS-0304062 is gratefully acknowledged.

\section{References}

1. Schmid G, Baumle M, Geerkens M, Helm I, Osemann C, Sawitowski T (1999) Current and future applications of nanoclusters. Chem Soc Rev 28(3):179-185

2. Curulli A, Valentini F, Padeletti G, Cusma A, Ingo GM, Kaciulis S, Caschera D, Palleschi G (2005) Gold nanotubules arrays as new materials for sensing and biosensing: synthesis and characterization. Sens Actuators B Chem 111:526-531

3. He B, Son SJ, Lee SB (2006) Shape-coded silica nanotubes for biosensing. Langmuir 22(20):8263-8265

4. Coker EN, Steen WA, Miller JT, Kropf AJ, Miller JE (2007) Nanostructured $\mathrm{Pt} / \mathrm{C}$ electrocatalysts with high platinum dispersions through zeolite-templating. Microporous Mesoporous Mater 101(3):440-444

5. Wang QB, Tang HP, Xi ZP, Zhang J, Li ZF (2006) The studies progress of the metal porous material using in the gasification technology. Rare Met Mater Eng 35:448-451

6. Choi JH, Ahn IS, Bak YC, Bae SY, Ha SJ, Jang HJ (2004) Preparation of high porous metal filter element for the fail-safety function. Powder Technol 140(1-2):98-105

7. Erlebacher J (2004) An atomistic description of dealloyingporosity evolution, the critical potential, and rate-limiting behavior. J Electrochem Soc 151(10):C614-C626

8. Yang Y, Matsubara S, Nogami M, Shi JL, Huang WM (2006) One-dimensional self-assembly of gold nanoparticles for tunable surface plasmon resonance properties. Nanotechnology 17 (11):2821-2827

9. Ding Y, Kim YJ, Erlebacher J (2004) Nanoporous gold leaf: "Ancient technology"/advanced material. Adv Mater 16(21):1897
10. Yu F, Ahl S, Caminade AM, Majoral JP, Knoll W, Erlebacher J (2006) Simultaneous excitation of propagating and localized surface plasmon resonance in nanoporous gold membranes. Anal Chem 78(20):7346-7350

11. Thompson WR, Pemberton JE (1995) Thin sol-gel silica films on (3-Mercaptopropyl)Trimethoxysilane-modified Ag And Au Surfaces. Chem Mater 7(1):130-136

12. Yu F, Yao DF, Knoll W (2003) Surface plasmon field-enhanced fluorescence spectroscopy studies of the interaction between an antibody and its surface-coupled antigen. Anal Chem 75 (11):2610-2617

13. Spinke J, Liley M, Guder HJ, Angermaier L, Knoll W (1993) Molecular recognition at self-assembled monolayers - the construction of multicomponent multilayers. Langmuir 9(7):1821-1825

14. Angersteinkozlowska H, Conway BE, Hamelin A, Stoicoviciu L (1986) Elementary steps of electrochemical oxidation of singlecrystal planes of $\mathrm{Au}$.1. Chemical basis of processes involving geometry of anions and the electrode surfaces. Electrochim Acta 31(8):1051-1061

15. Bonroy K, Friedt JM, Frederix F, Laureyn W, Langerock S, Campitelli A, Sara M, Borghs G, Goddeeris B, Declerck P (2004) Realization and characterization of porous gold for increased protein coverage on acoustic sensors. Anal Chem 76(15):4299-4306

16. Jurczakowski R, Hitz C, Lasia A (2004) Impedance of porous Au based electrodes. J Electroanal Chem 572(2):355-366

17. Dahlin A, Zach M, Rindzevicius T, Kall M, Sutherland DS, Hook F (2005) Localized surface plasmon resonance sensing of lipidmembrane-mediated biorecognition events. J Am Chem Soc 127 (14):5043-5048

18. Cui XQ, Pei RJ, Wang XZ, Yang F, Ma Y, Dong SJ, Yang XR (2003) Layer-by-layer assembly of multilayer films composed of avidin and biotin-labeled antibody for immunosensing. Biosens Bioelectron 18(1):59-67

19. Liu J (2005) Systematic studies of protein immobilization by surface plasmon field-enhanced fluorescence spectroscopy. PhD Thesis, University of Mainz 